PREPARED TO BE SUBMITTED To the Astrophysical Journal

Preprint typeset using LTEX style emulateapj v. 5/2/11

\title{
A VIRIAL CORE IN THE SCULPTOR DWARF SPHEROIDAL GALAXY
}

\author{
A. Agnello and N. W. Evans \\ Institute of Astronomy, University of Cambridge, Madingley Road, Cambridge CB3 OHA, UK \\ Draft version September 24, 2018
}

\begin{abstract}
The projected virial theorem is applied to the case of multiple stellar populations in the nearby dwarf spheroidal galaxies. As each population must reside in the same gravitational potential, this provides strong constraints on the nature of the dark matter halo. We derive necessary conditions for two populations with Plummer or exponential surface brightnesses to reside in a cusped Navarro-Frenk-White (NFW) halo. We apply our methods to the Sculptor dwarf spheroidal, and show that there is no NFW halo compatible with the energetics of the two populations. The dark halo must possess a core radius of $\sim 120 \mathrm{pc}$ for the virial solutions for the two populations to be consistent. This conclusion remains true, even if the effects of flattening or self-gravity of the stellar populations are included.

Subject headings: Galaxies: dwarf - Galaxies: individual: Sculptor - Galaxies: kinematics and dynamics - dark matter
\end{abstract}

\section{INTRODUCTION}

Using high resolution numerical simulations of structure formation, Navarro, Frenk \& White (1995) first proposed that dark haloes have the universal form

$$
\rho(r)=\frac{\rho_{0}}{\left(r / r_{\mathrm{s}}\right)\left(1+r / r_{\mathrm{s}}\right)^{2}}
$$

where $r_{s}$ is the scale-radius and $\rho_{0}$ is the density normalisation. Sometimes it is useful to define a halo radius $r_{\mathrm{h}}$, which is the radius within which the mean density is a factor of $\Delta_{\mathrm{h}}=200$ times greater than the critical density. Then, the enclosed mass of the NFW profile is

$$
M(r)=4 \pi \rho_{0} r_{\mathrm{s}}^{3}\left[\log (1+c x)-\frac{c x}{1+c x}\right]
$$

where $x=r / r_{\mathrm{h}}$ and $c=r_{\mathrm{h}} / r_{\mathrm{s}}$. The two parameters of the NFW halo can be alternatively chosen as the concentration $c$ and the mass $M=M\left(r_{\mathrm{h}}\right)$. The NFW model remains the best two-parameter fit to simulation data, though Navarro et al. (2004) provided evidence that the three-parameter Einasto profile gives a still better match.

Despite an extensive body of research, it is unclear whether the shapes of dark haloes inferred from observations are consistent with cosmological predictions. In galaxies like the Milky Way and M31, the dark halo profiles have been significantly affected by the cooling of baryons, which makes them unattractive candidates for testing the prediction. Therefore, research has concentrated on two very different classes of objects, namely galaxy clusters and dwarf galaxies. In clusters, the high temperature and low density of the intra-cluster gas prevents efficient cooling, and so the baryons do not significantly affect the potential of the dark matter. There is reasonably good evidence from cluster lensing that the profiles follow the NFW law, although with higher concentrations than predicted (e.g., Broadhurst et al. 2008).

In dwarf galaxies at the present epoch, the baryonic component is feeble in comparison with the dark matter. Low surface brightness galaxies with $\mathrm{HI}$ and $\mathrm{H} \alpha$ rotation curves have been studied extensively by de Blok \& Bosma (2002), who

aagnello@ast.cam.ac.uk (AA), nwe@ast.cam.ac.uk (NWE) conclude that cored rather than cusped haloes are favoured. The dwarf spheroidal galaxies have no gas, and are pressuresupported rather than rotation-supported, so are more difficult to assess. It was quickly realised that the radial velocities of thousands of bright giant stars could be gathered with modern multi-object spectrographs (Kleyna et al. 2002; Walker et al. 2007). Dynamical modelling with either the Jeans equations or phase space distribution functions then yields constraints on the dark matter profile. However, this line of inquiry seemed to have stalled with the realization of the degeneracies inherent in the Jeans equations, making the datasets compatible with both cored and cusped dark halo models depending on the underlying assumptions (Evans et al. 2009; Walker et al. 2009).

The problem has received new impetus with the growing realization that dwarf spheroidals often contain two or more stellar populations (Battaglia et al. 2006; Koch et al. 2006). This significantly strengthens any constraints from dynamical modelling, as now each sub-population must separately be in equilibrium in the same potential. There have been three recent and thought-provoking results. First, Battaglia et al. (2008) used the Jeans equations to model the velocity dispersion profiles of the two populations in the Sculptor dSph, finding that a cored halo is preferred to an NFW halo, though the latter is still statistically consistent with the observations. Second, using the same dataset but more rigorous methods based on distribution functions, Amorisco \& Evans (2012a) showed that NFW halos are rejected at high significance, performing substantially poorer than cored models. Finally, Walker \& Peñarrubia (2011) used the half-light radii of the two populations in the Fornax and Sculptor dSphs to estimate the enclosed mass. This exploited earlier results showing that the mass within the half-light radius is robust against changes in the anisotropy (Walker et al. 2009; Wolf et al. 2010). Given the enclosed mass at two radii, the slope of density is inferred and shown to be in contradiction with NFW haloes.

Here, we provide a simple line of reasoning based on the energies of the subpopulations. This brings the earlier arguments of Battaglia et al. (2008), Walker \& Peñarrubia (2011) and Amorisco \& Evans (2012a) into sharp focus. We show that the energetics of the subpopulations in one of the best studied dSphs, Sculptor, cannot be made consistent with an 
NFW halo.

\section{THE PROJECTED VIRIAL THEOREM}

Let $z$ be the line of sight direction. Then, the projected virial theorem relates the projected component of the pressure and potential energy tensors by the equation $2 K_{\text {los }}+W_{\text {los }}=0$, where

$$
K_{\mathrm{los}}=\frac{\Upsilon_{\star}}{2} \int v\left\langle v_{\mathrm{z}}^{2}\right\rangle \mathrm{d}^{3} x, \quad W_{\mathrm{los}}=-\int v z \partial_{z} \Phi \mathrm{d}^{3} x,
$$

Here, $v$ is the luminosity density of a population with stellar mass-to-light ration $\Upsilon_{\star}$ moving in the gravity field $\Phi$. Under the assumption of spherical symmetry, the tensors become (e.g., Merrifield \& Kent 1990)

$$
\begin{aligned}
K_{\mathrm{los}} & =\pi \Upsilon_{\star} \int_{0}^{\infty} \mu\left\langle v_{\mathrm{los}}^{2}\right\rangle R \mathrm{~d} R \\
W_{\mathrm{los}} & =-\frac{4 \pi G}{3} \Upsilon_{\star} \int_{0}^{\infty} r \nu(r) M(r) \mathrm{d} r
\end{aligned}
$$

where $M(r)$ is the mass enclosed within radius $r$. These formulae involve the line of sight velocity second moment $\left\langle v_{\text {los }}^{2}\right\rangle$ and the surface brightness $\mu$, which are directly accessible to observation. Notice that the velocity anisotropy of the stellar population does not enter, which gives the virial equations an immediate advantage over the Jeans equations. Notice also that virial quantities, which are gross volume integrals, can be computed more robustly than the gradients of observables required by the Jeans equations. Furthermore, there is no problem in dealing with rotation, as the integral $K_{\text {los }}$ requires just the sum of square velocities in ordered and random motions along the line of sight.

The only unknown in the projected virial equation is the total gravitational potential $\Phi(r)$ in which the population moves, or equivalently the enclosed mass $M(r)$. If preferred, $W_{\text {los }}$ can be recast in terms of the total mass density $\rho(r)$ which generates $\Phi$, namely

$$
W_{\mathrm{los}}=-\frac{16 \pi G}{3} \Upsilon_{\star} \int_{0}^{\infty} R \mu(R) \int_{0}^{R} \rho(r) \frac{r^{2} \mathrm{~d} r}{\sqrt{R^{2}-r^{2}}} \mathrm{~d} r \mathrm{~d} R .
$$

Here, we have eliminated the luminosity density in terms of the surface brightness, which is an observable.

The projected virial equation by itself is of course insufficient to determine the potential or mass distribution directly. Suppose now there are two populations in equilibrium in the same total potential (e.g., Agnello \& Evans 2012). In the dSphs, a younger metal-rich and an older metal-poor population with associated velocity dispersion profiles $\sigma_{\mathrm{r}}$ and $\sigma_{\mathrm{p}}$ and surface brightnesses $\mu_{\mathrm{r}}$ and $\mu_{\mathrm{p}}$ (with corresponding luminosity densities $v_{\mathrm{r}}$ and $\nu_{\mathrm{p}}$ ) are often present. For example, there is good evidence that Sculptor (Battaglia et al. 2008; Walker \& Peñarrubia 2011), Carina (Bono et al. 2010) and Fornax (Amorisco \& Evans 2012b) contain at least two distinct populations. Then, the projected virial theorem must be satisfied for each population, so we have

$$
\frac{K_{\mathrm{los}, \mathrm{p}}}{K_{\mathrm{los}, \mathrm{r}}}=\frac{W_{\mathrm{los}, \mathrm{p}}}{W_{\mathrm{los}, \mathrm{r}}} \Longrightarrow \frac{\int_{0}^{\infty} \mu_{\mathrm{p}} \sigma_{\mathrm{p}}^{2} d R}{\int_{0}^{\infty} \mu_{\mathrm{r}} \sigma_{\mathrm{r}}^{2} d R}=\frac{\int_{0}^{\infty} v_{\mathrm{p}} M(r) r \mathrm{~d} r .}{\int_{0}^{\infty} v_{\mathrm{r}} M(r) r \mathrm{~d} r .}
$$

The only unknown is the potential, or equivalently, the enclosed mass $M(r)$. This result is powerful enough to rule out some dark matter haloes. The physical reason is that a stellar population with velocity dispersion $\left\langle v_{\text {los }}^{2}\right\rangle$ offers the best constraint on the potential at radii given by $\Phi(r) \approx\left\langle v_{\text {los }}^{2}\right\rangle$. With more than one populations, the potential is restricted at more than one location.

\section{APPLICATION TO SCULPTOR}

\subsection{Simple Results}

If the potential is heavily dominated by dark matter, we can neglect the self-gravity of the luminous components in a first approximation (relaxed in the next sub-section). In this case, both the kinetic energy tensor $K_{\text {los }}$ and the potential energy tensor $W_{\text {los }}$ scale linearly with the stellar mass-to-light ratio, which can then be eliminated.

The surface brightness profiles of dSphs are well-fit by Plummer laws

$$
\mu(R)=\frac{\mu_{0}}{\left(1+R^{2} / R_{\mathrm{h}}^{2}\right)^{2}} \quad .
$$

This assumption is not critical to our argument, but it does provide a useful illustrative model. We shall in any case sketch the extension to another commonly used profile, namely the exponential law. Fitting the Plummer law to the profiles of the metal-rich and metal-poor populations in Sculptor gives $R_{\mathrm{h}, \mathrm{r}}=230 \pm 10$ pc for the metal-rich and and $R_{\mathrm{h}, \mathrm{p}}=350 \pm 10 \mathrm{pc}$ for the metal-poor (consistent with the values in Battaglia et al. (2008) and Amorisco \& Evans (2012a)). A convenient fitting formula for the velocity dispersion profile is

$$
\left\langle v_{\mathrm{los}}^{2}\right\rangle=\frac{\sigma_{0}^{2}}{\left(1+R^{2} / a^{2}\right)},
$$

which gives a flattish profile with amplitude $\sigma_{0}$ out to a characteristic lengthscale $a$. This is typical of the velocity dispersion profiles in, for example, Walker et al. (2007) or Battaglia et al. (2008). Again, this assumption is not necessary for our argument, as the relevant virial integrals can always be computed numerically. For Sculptor, fits provide $\sigma_{0, \mathrm{r}}=8.7 \pm 1.0 \mathrm{kms}^{-1}$ and $a_{\mathrm{r}}=240 \pm 55 \mathrm{pc}$ for the metal-rich and $\sigma_{0, \mathrm{p}}=10.9 \pm 0.8 \mathrm{kms}^{-1}$ and $a_{\mathrm{p}}=1920 \pm 850 \mathrm{pc}$ for the metal-poor.

With these laws, the kinetic energy integral is analytic with

$$
K_{\mathrm{los}}=\frac{\pi}{2} \Upsilon_{\star} \mu_{0} \sigma_{0}^{2} a^{2} \frac{a^{2} / R_{\mathrm{h}}^{2}-1-2 \log \left(a / R_{\mathrm{h}}\right)}{\left(1-a^{2} / R_{\mathrm{h}}^{2}\right)^{2}} .
$$

The metal-rich limit is given by $a_{\mathrm{r}} \approx R_{\mathrm{h}, \mathrm{r}}$, the metal-poor limit is given by $a_{\mathrm{p}} \gg R_{\mathrm{h}, \mathrm{p}}$. This gives

$$
\frac{K_{\mathrm{los}, \mathrm{p}}}{K_{\mathrm{los}, \mathrm{r}}}=2\left(\frac{\mu_{0, \mathrm{p}}}{\mu_{0, \mathrm{r}}}\right)\left(\frac{R_{\mathrm{h}, \mathrm{p}}}{R_{\mathrm{h}, \mathrm{r}}}\right)^{2}\left(\frac{\sigma_{0, \mathrm{p}}}{\sigma_{0, \mathrm{r}}}\right)^{2} .
$$

The projected potential energy of Plummer profiles embedded in NFW halos is also exact, as

$$
\begin{aligned}
W_{\text {los }} & =-\frac{8 \pi^{2} G \Upsilon_{\star} \mu_{0} \rho_{0} R_{\mathrm{h}}^{3} r_{\mathrm{s}}^{3}}{3 \Delta^{5}}\left[\Delta\left(r_{\mathrm{s}}^{2}+3 r_{\mathrm{s}} R_{\mathrm{h}}-2 R_{\mathrm{h}}^{2}\right)\right. \\
& \left.+R_{\mathrm{h}}\left(R_{\mathrm{h}}^{2}-2 r_{\mathrm{s}}^{2}\right) \log \left(\frac{R_{\mathrm{h}}\left(\Delta+R_{\mathrm{h}}\right)}{r_{\mathrm{s}}\left(\Delta-r_{\mathrm{s}}\right)}\right)\right]
\end{aligned}
$$

with $\Delta^{2}=R_{\mathrm{h}}^{2}+r_{\mathrm{s}}^{2}$. With the values of $R_{\mathrm{h}}$ listed above, the virial ratio $W_{\text {los,p }} / W_{\text {los,r }}$ as a function of $r_{\mathrm{s}}$ is increasing and bound from above. In particular, we have that

$$
\frac{W_{\text {los }, \mathrm{p}}}{W_{\text {los }, \mathrm{r}}}<\left(\frac{\mu_{0, \mathrm{p}}}{\mu_{0, \mathrm{r}}}\right)\left(\frac{R_{\mathrm{h}, \mathrm{p}}}{R_{\mathrm{h}, \mathrm{r}}}\right)^{3} .
$$



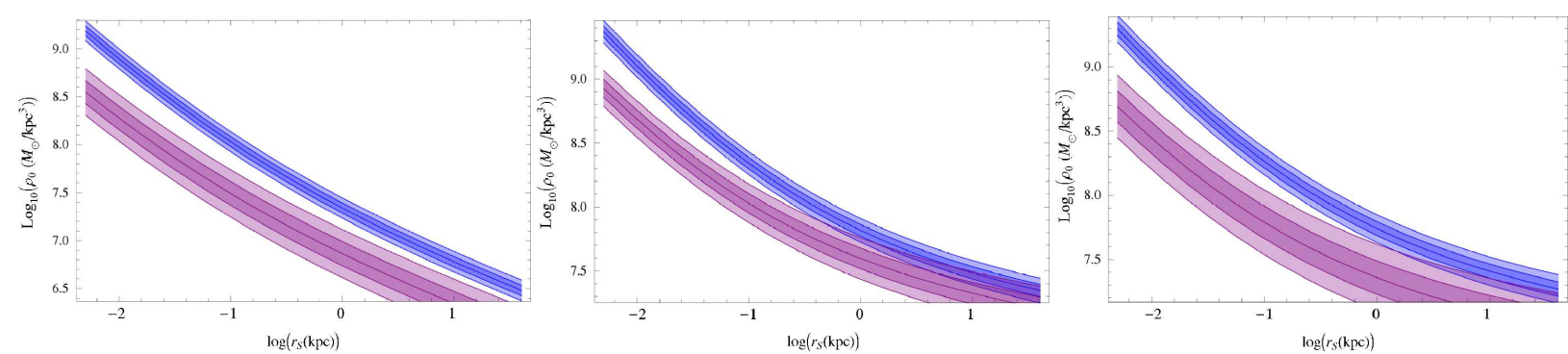

Figure 1. Left: Virial stripes for the two stellar populations in Sculptor in a cusped NFW potential, including the self-gravity of the stellar populations $\left(\Upsilon_{\star}=8\right)$. Purple shows the metal-rich population, blue the metal-poor population. In each stripe, the central line is the mean value of $\log _{10}\left(\rho_{0}\right)$, whilst the median and outer lines follow the $1 \sigma$ and $2 \sigma$ deviations. Center and Right: Virial stripes for the two stellar populations in a NFW potential with small core, without $\left(\Upsilon_{\star}=0\right)$ and with $\left(\Upsilon_{\star}=8\right)$ self-gravity.

\begin{tabular}{|l|c|c|c|}
\hline$\epsilon=r_{\mathrm{c}} / r_{\mathrm{S}}$ & $\begin{array}{c}r_{\mathrm{s}} \text { [in kpc] } \\
\left(\Upsilon_{\star} \rightarrow 0\right)\end{array}$ & $\begin{array}{c}r_{\mathrm{s}} \text { [in kpc] } \\
\left(\Upsilon_{\star}=4\right)\end{array}$ & $\begin{array}{c}r_{\mathrm{s}} \text { [in kpc] } \\
\left(\Upsilon_{\star}=8\right)\end{array}$ \\
\hline 1 & 0.72 & 1.06 & 1.23 \\
0.5 & 0.94 & 1.40 & 1.54 \\
0.25 & 1.2 & 1.92 & 2.20 \\
0.125 & 1.6 & 2.88 & 3.28 \\
0.0625 & 2.4 & 4.48 & 4.96 \\
\hline
\end{tabular}

Table 1

Minimum $r_{\mathrm{s}}$ for two-sigma overlapping of the virial stripes.

Hence, a necessary condition for a NFW halo to support two stellar populations with Plummer profiles is

$$
\left(\frac{\sigma_{0, \mathrm{r}}}{\sigma_{0, \mathrm{p}}}\right)^{2}>2\left(\frac{R_{\mathrm{h}, \mathrm{r}}}{R_{\mathrm{h}, \mathrm{p}}}\right) .
$$

This is identical to eq (22) of Amorisco \& Evans (2012a), derived under different assumptions. If, instead of Plummer profiles, exponential laws are used to fit the surface brightness profiles, then the numerical factor becomes 1.9 instead of 2 in eqn (11). The analogue of eqn (13) is unchanged, so that the necessary condition for an NFW halo to support two stellar populations with exponential surface brightness profiles is

$$
\left(\frac{\sigma_{0, \mathrm{r}}}{\sigma_{0, \mathrm{p}}}\right)^{2}>1.9\left(\frac{R_{\mathrm{h}, \mathrm{r}}}{R_{\mathrm{h}, \mathrm{p}}}\right) \text {. }
$$

Using the best-fitting values provided above for Sculptor, it is immediate to check that the NFW potential is ruled out. Note that the constraints are simply the requirement that there is a NFW model with $r_{\mathrm{s}}<\infty$. This is a much looser constraint than requiring consistency with an NFW model with a concentration $c \approx 20$, as predicted by cold dark matter theories.

\subsection{The Virial Stripes}

The simple results already suggest that the energetics of the two populations are inconsistent with an NFW profile. However, it is prudent to confirm this result numerically, discarding some of the simplifying assumptions made above.

Since the measured profiles come with errors, we operate in the following manner. For each value of $r_{\mathrm{s}}$, we compute $\rho_{0}$ separately for the two populations for many different photometric (8) and kinematic (9) profiles. We weight each result with the likelihood of the fit. Then, varying $r_{\mathrm{s}}$ produces a virial stripe for each population in the $\left(\rho_{0}, r_{\mathrm{s}}\right)$-plane. If the two stripes overlap at $2 \sigma$ at a particular $r_{\mathrm{s}}$, then the model for the potential is plausible at the $2 \sigma$ level. Nothing prevents us from including the contribution of the luminous tracers to the potential as well. The virial equations then depend also on the stellar mass-to-light ratio $\Upsilon_{\star}$, which may be different for the two populations. The projected potential energy $W_{\text {los }}$ has a contribution $W_{\mathrm{dm}}$ from the dark component and a correction $W_{\text {self }}$ from the two luminous ones. For Plummer profiles, we have for the $i$-th population:

$$
W_{\text {self }, i}=\pi^{2} G \mu_{0, i} R_{h, i} \sum_{j} \Upsilon_{\star, j} \mu_{0, j} R_{h, j}^{2} w\left(R_{\mathrm{h}, i} / R_{\mathrm{h}, j}\right),
$$

with

$$
w(x)=\frac{x^{3}\left[\left(5 x^{2}+3\right) \mathrm{K}\left(1-x^{2}\right)-\left(x^{2}+7\right) \mathrm{E}\left(1-x^{2}\right)\right]}{3\left(1-x^{2}\right)^{3}},
$$

where $\mathrm{K}, \mathrm{E}$ are complete elliptic integrals and $\Upsilon_{\star, j}$ is the luminous mass-to-light ratio of the $j$-th population. As the $\mu_{0, j}$ are given by number counts and not directly by luminosities, a common rescaling is applied to both populations such that the total luminosity is fixed at the observed value (taken from table 6 in Irwin \& Hatzidimitriou 1995).

The leftmost panel of Figure 1 shows the virial stripes for the two populations in Sculptor, excluding and including the effects of the self-gravity for the luminous component. The two stripes never overlap at the $2 \sigma$ level. This confirms the result deduced from our simple argument in the previous section: there is no NFW halo compatible with the kinetic energies of the two stellar components in Sculptor. The center and rightmost panels of Figure 1 show the virial stripes when the dark halo density is the simplest cored analogue of the NFW halo, namely

$$
\mathrm{cNFW}=\frac{\rho_{0}}{\left(\epsilon^{2}+r^{2} / r_{\mathrm{s}}^{2}\right)^{1 / 2}\left(1+r^{2} / r_{\mathrm{s}}^{2}\right)} .
$$

In this case, the stripes do overlap at the $2 \sigma$ level provided the core radius $r_{\mathrm{c}} \equiv r_{\mathrm{s}} \epsilon$ is at least $150 \mathrm{pc}$, if the self-gravity of the stellar populations is neglected. Incorporating self-gravity causes the core radius to increase somewhat, as we see in Table 1. This shows how the minimum $r_{\mathrm{s}}$ for $2 \sigma$ overlap varies with changing $\Upsilon_{\star}$ for different models. The first column $\left(\Upsilon_{\star} \rightarrow 0\right)$ stands for models in which the self-gravity of the stars is omitted. Since both half-light radii are smaller or equal to $r_{\mathrm{s}}$ in the dark matter only case, adding self-gravity is expected to yield larger cores, as in fact is confirmed by the results in the Table.

\section{DISCUSSION AND CONCLUSIONS}

The arguments in this Letter show that the kinematics of multiple populations in $\mathrm{dSphs}$ provide a substantial challenge to the predictions of cold dark matter cosmogonies. In the case of one of the best studied dSphs, Sculptor, there is no 


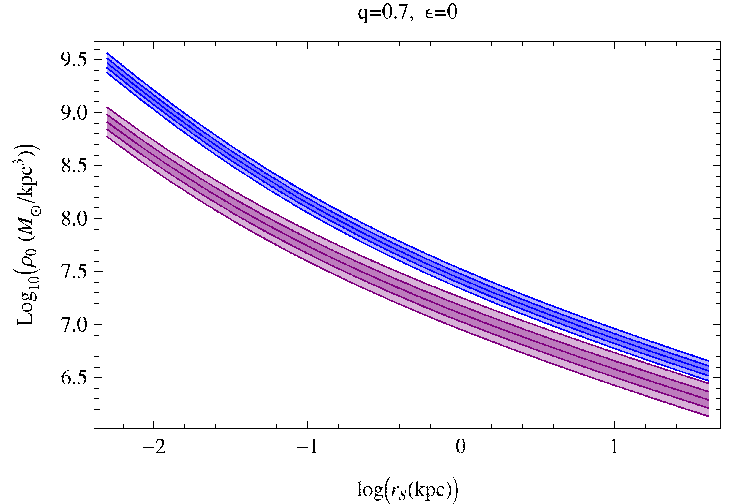

Figure 2. Virial stripes for the two stellar populations in Sculptor in a flattened NFW potential $\left(q / g=0.7\right.$ with $\left.\Upsilon_{\star}=0\right)$. Purple shows the metal-rich population, blue the metal-poor population. In each stripe, the central line is the mean value of $\log _{10}\left(\rho_{0}\right)$, whilst the median and outer lines follow the $1 \sigma$ and $2 \sigma$ deviations.

NFW dark halo that is compatible with the available photometric and kinematics data. The problem is very basic - the energetics of the metal-rich and metal-poor populations do not permit them to reside in the same NFW halo. Are there are any loop-holes?

\subsection{Reliability of Separation of Sub-Populations?}

This Letter has used the separation proposed by Battaglia et al. (2008), who used a hard cut in metallicity to define the metal-poor $([\mathrm{Fe} / \mathrm{H}]<-1.7)$ and metal-rich populations $([\mathrm{Fe} / \mathrm{H}]>-1.5)$. This is open to the objection that the kinematics and metallicity are interlocked, and so the cut may be subject to unperceived biases. The sub-populations can also be separated using a maximum likelihood approach with metallicity and kinematics treated jointly. We have checked our calculations using such a method on the sample of stars in Tolstoy et al. (2004) (also used by Battaglia et al. 2008). Walker \& Peñarrubia (2011) have also tacked this problem, using a Bayesian likelihood estimator based on positional, kinematical and chemical data to separate the subpopulations. Their algorithm also makes assumptions that may or may not be warranted (for example, the velocity distributions are assumed isothermal). Nonetheless, although the details of the separation are different, Walker \& Peñarrubia (2011) come to the same conclusion that the multiple populations of Sculptor are inconsistent with an NFW halo. Different methods of separation, using different algorithms, come to the same answer, which offers comfort to those concerned about reliability.

\subsection{Steady state, well mixed populations?}

An assumption in the virial theorem is that the stellar populations are in a steady-state and well-mixed. Recently, de Boer et al (2012) have provided a star-formation history for Sculptor. There was a peak in star formation $\sim 13-14 \mathrm{Gyr}$ ago, providing the bulk of the old, metal-poor population. Star formation continued till $\tau_{0} \sim 7$ Gyr ago. With the typical dark matter densities found from the virial stripes, the dynamical time-scale $\left(4 \pi G \rho_{0}\right)^{-1 / 2}$ is at least one order of magnitude smaller than $\tau_{0}$. It seems that the two populations have indeed reached a steady-state and that each of them is internally well-mixed. From the Hubble Space Telescope proper motions, the orbital period of Sculptor is estimated as $\sim 2.2 \mathrm{Gyr}$ (Piatek et al. 2006). Its pericentric distance is $\sim 68 \mathrm{kpc}$, and the time of the last pericentric passage was $\sim 1$ Gyr ago. As the dSph always remains in the outer parts of the Milky Way halo, it seems very improbable that tidal stirring can overturn the steady-state hypothesis.

\subsection{Flattening?}

A drawback of all previous models of dSphs is that they are restricted to spherical symmetry. This is not the case for our virial arguments.

The isophotes of the Sculptor dSph have a measured ellipticity of 0.3 (Irwin \& Hatzidimitriou 1995). In fact, the flattening of the dark matter potential must be rounder than the flattening of the luminosity density, so spherical models are probably a good approximation. As a check, we generalize our virial arguments to flattened stellar populations (with axis ratio $q$ ) in a flattened NFW dark matter halo (with axis ratio $g$ ). Let us assume that Sculptor is viewed edge-on,and the density and kinematics of both stellar populations are stratified on surfaces $x^{2}+y^{2} q^{-2}$. We also make the natural assumption that the minor axes of the dark matter and the luminous matter are aligned. Then, each kinetic energy term in eqn (7) scales by $q$, and so the ratio is left unchanged. Similarly, each potential energy term in eqn (7) is modulated by the same function of $q / g$ in the integrand (c.f., the Flattening Theorem of Agnello \& Evans 2012) and so the ratio is left largely unchanged. This suggests that our virial arguments will be robust against flattening.

To confirm this, we carried out explicit calculations of the virial stripes for flattened models of Sculptor with $q / g=0.7$, an example of which is shown in Fig 2. The virial stripes for the flattened NFW halo never overlap. Introducing a small core does permit the kinematics of the two populations to be consistent at the $2 \sigma$ level providing the core is $\gtrsim 120 \mathrm{pc}$, a touch smaller than in the spherical case.

We believe that the virial arguments are particularly robust and emphasise the important point that the energetics of the two populations in Sculptor are completely incompatible with a $1 / r$ dark matter density cusp. For at least this dwarf galaxy, we can securely state that it either never had a $1 / r$ density cusp, or if it did have one, feedback processes have now removed it.

Of course, there has been much recent interest in converting cusped haloes into cored. A number of promising possibilities have been suggested, including impulsive mass loss from supernovae (Read \& Gilmore 2005), winds and gas flows driven by supernovae (Mashchenko et al. 2006) and infalling baryonic clumps (Cole et al. 2011). Which of these processes, if any, were responsible for the core in Sculptor is an important question for future work.

AA thanks the Science and Technology Facility Council and the Isaac Newton Trust for the award of a studentship. We wish to thank Nicola C. Amorisco and the anonymous referee for critical readings of the manuscript, and Giuseppina Battaglia and Mike Irwin for generously providing us with data.

\section{REFERENCES}

Agnello A., Evans N.W. 2012, MNRAS, 422, 1767

Amorisco N., Evans N.W., 2012a, MNRAS, 419, 184

Amorisco N., Evans N.W., 2012b, in preparation.

Battaglia, G., Tolstoy, E., Helmi, A., et al. 2006, A\&A, 459, 423 
Battaglia, G., Helmi, A., Tolstoy, E., et al. 2008, ApJ, 681, L13

Bono, G., Stetson, P. B., Walker, A. R., et al. 2010, PASP, 122, 651

Broadhurst, T., Umetsu, K., Medezinski, E., Oguri, M., \& Rephaeli, Y. 2008, ApJ, 685, L9

de Blok, W. J. G., \& Bosma, A. 2002, A\&A, 385, 816

de Boer, T. J. L., Tolstoy, E., Hill, V., et al. 2012, A\&A, 539, A103

Cole, D. R., Dehnen, W., \& Wilkinson, M. I. 2011, MNRAS, 416, 1118

Evans, N. W., An, J., Walker, M. G. 2009, MNRAS, 393, L50

Irwin M.J., Hatzidimitriou D. 1995, MNRAS, 277, 1354

Kleyna, J., Wilkinson, M. I., Evans, N. W., Gilmore, G., Frayn, C. 2002, MNRAS, 330, 792

Koch, A., Grebel, E. K., Wyse, R. F. G., et al. 2006, AJ, 131, 895

Mashchenko, S., Couchman, H. M. P., \& Wadsley, J. 2006, Nature, 442, 539
Merrifield, M. R., Kent, S. M. 1990, AJ, 99, 15481

Navarro, J. F., Frenk, C. S., \& White, S. D. M. 1995, MNRAS, 275, 720

Navarro, J. F., Hayashi, E., Power, C., et al. 2004, MNRAS, 349, 1039

Piatek, S., Pryor, C., Bristow, P., et al. 2006, AJ, 131, 1445

Read, J. I., \& Gilmore, G. 2005, MNRAS, 356, 107

Tolstoy, E., et al. 2004, ApJ, 617, L119

Walker, M. G., Mateo, M., Olszewski, E. W., et al. 2007, ApJ, 667, L53

Walker, M. G., Mateo, M., Olszewski, E. W., et al. 2009, ApJ, 704, 1274

Walker, M. G., \& Peñarrubia, J. 2011, ApJ, 742, 20

Wolf, J., Martinez, G. D., Bullock, J. S., Kaplinghat, M., Geha, M., Muñoz,

R. R., Simon, J. D., Avedo, F. F. 2010, 